\title{
An Improved Data Error Detection Technique for Dependable Embedded Software
}

\author{
Venu Babu Thati, Jens Vankeirsbilck, Jeroen Boydens \\ Dept. of Computer Science \\ KU Leuven Bruges Campus \\ Spoorwegstraat 12, \\ 8200 Brugge, Belgium \\ Venubabu.Thati@kuleuven.be
}

\author{
Davy Pissoort \\ Dept. of Electrical Engineering \\ KU Leuven Bruges Campus \\ Spoorwegstraat 12, \\ 8200 Brugge, Belgium \\ Davy.Pissoort@kuleuven.be
}

\author{
Niels Penneman \\ Televic Healthcare $N \mathrm{~V}$ \\ Leo Bekaertlaan 1, \\ 8870 Izegem, Belgium \\ N.Penneman@televic.com
}

\begin{abstract}
This paper presents a new software-implemented data error detection technique called Full Duplication and Selective Comparison. Our technique combines the ideas of existing techniques in order to increase the fault detection ratio, decrease the imposed code size and execution time overhead. As the name gives away, we opt to duplicate the entire code base and place comparison instructions in critical basic blocks only. The critical basic blocks are the blocks with two or more incoming edges. We evaluate our technique by implementing it for several case studies and by performing fault injection experiments. Next, we compared the obtained results to the parameters of three established techniques: Error Detection by Diverse Data and Duplicated Instructions, Critical Block Duplication and Software Implemented Fault Tolerance. The results show an average increase of $20.5 \%$ in fault detection ratio and an average decrease in code size and execution time overhead of $12.6 \%$ and $0.5 \%$, respectively.
\end{abstract}

Keywords-data error detection, reliability, fault tolerance, bit-flips, instruction duplication

\section{INTRODUCTION}

In recent years, embedded systems are being increasingly used in daily life. They are commonly used in mechatronics, aerospace and medical applications due to the low cost and the adaptable software solutions they provide. At the same time, embedded systems are intrinsically susceptible to bit-flips. This is mainly because of a reduction in the size of components and increasing complexity of the latest computer architectures [1]-[3]. Generally, these bitflips occur as a result of many environmental factors such as electromagnetic interference (EMI), high-energy particle radiation [4] and temperature changes [5]. Unlike production and design faults, bit-flips or soft errors do not occur constantly and cannot be anticipated without fault-tolerant mechanisms. These are also called transient faults. Transient faults do not cause permanent physical damage to the system and can be recovered by overwriting the introduced bit-flip or restarting the system [6].

Bit-flips in embedded systems can corrupt the reliability and integrity of the system. According to recent studies, bitflips are one of the immediate sources of failure in embedded systems [7]-[9]. Such bit-flips have a further effect on the program during execution. The effect can be mainly in terms of data errors and control flow errors. Data errors may lead to corruption of variables in the program causing wrong intermediate and output results. On the other hand, control flow errors may lead to a jump in the program execution order, causing a system to hang or crash [1], [10]-[12]. As like data errors, control flow errors can also lead to wrong outputs. Therefore, it is important for researchers, designers and developers to make embedded systems faulttolerant against bit-flips.

Fault tolerance can be achieved via hardware- or softwarebased solutions [13], [14]. Typical hardware-based solutions are hardware redundancy techniques to achieve reliability. Techniques based on hardware redundancy provide good fault coverage with additional cost. To overcome the additionally imposed overhead and cost in hardware-based techniques, many different redundancies have been proposed and used [15]-[17]. Among them, software redundancy is a popular one [13], [18]. Despite the fact that softwarebased redundancy techniques provide less fault detection than hardware techniques, by implementing redundancy techniques in software, it is a one-time engineering cost at the beginning of the project. In addition, an overall lower price can be achieved. Unlike hardware-based techniques, software-based ones are flexible enough and cost-effective to use in a wide range of applications.

Moreover, it is important to have a method that satisfies the requirement of fault detection ratio with a minimal overhead for general purpose applications. This paper focuses on data error detection, for which multiple techniques have been proposed in the past. Duplication and then comparison are the basic mechanisms involved in most software-based data error detection techniques [1], [6], [13], [14], [17][19]. Here, the original instructions are duplicated and the results obtained from original and duplicated instructions are compared. If any mismatch between the results is detected, an error is reported.

In this paper, we propose a new data error detection technique that aims to improve fault detection ratio and decrease the imposed overhead. This is achieved by merging the principles of two different established techniques: error detection by diverse data and duplicated instructions $\left(E D^{4} I\right)[7]$ and 
critical block duplication (CBD) [18]. Although there exist numerous techniques in state-of-the-art, we selected these two techniques because they proved to be the best techniques based on our previous empirical study on numerous techniques [20]. To validate our technique, we performed fault injection experiments and compared it to three established techniques: $E D^{4} I, \mathrm{CBD}$ and software implemented fault tolerance (SWIFT) [6].

The remainder of this paper is organized as follows. In Section II, we give some general background considering data error detection techniques and explain the principles that enable $E D^{4} I, \mathrm{CBD}$ and SWIFT to detect data errors. Section III describes how we merge the ideas from $E D^{4} I$ and CBD to create our new Full Duplication and Selective Comparison (FDSC) technique. Section IV describes the experimental setup. Section V discusses the results. Section VI presents conclusions of this paper and our future work plans are described in Section VII.

\section{BACKGROUND AND PREVIOUS WORKS}

As discussed in the previous section, bit-flips can corrupt the program data during execution, leading to its wrong execution. To counter data corruption, different softwarebased data error detection techniques have been proposed in the past [8], [14], [18], [21], [22]. Table I shows an example of assembly-level unprotected code that is protected by a data error detection technique. The unprotected code, shown on the left-hand side, uses 5 registers: $r 0$ to $r 4$. This means that the protected code, shown in the right column, has to use 10 registers, one duplicate for each original register. The duplicates are indicated with the '-symbol. In addition to duplicate instruction, comparison instructions have to be inserted to validate the results of both instructions. Furthermore, as mentioned in Table I, add, ldr, str, mov and cmp, indicates the assembly-level instructions of addition, load, store, move and compare operations, respectively. Likewise, ble, represents a branch instruction which is less or equal to a condition, whereas bne represents a branch instruction which is not equal to a condition.

Data error detection techniques presented in the literature are further classified into two categories: full code duplication and selective code duplication techniques [14]. The categorization is based on their duplication mechanisms.

\section{A. Full Code Duplication}

In full code duplication techniques, the entire code base is duplicated and their results are compared. This results in good fault detection ratio; however, they impose code size and execution time overhead of about $255 \%$ to the system [8]. To compare our Full Duplication and Selective Comparison (FDSC) technique, we choose $E D^{4} I$ from this full code duplication category. We selected this because it proved to be the best technique for fault detection ratio among other full code duplication techniques from our empirical study [20].

In $E D^{4} I$, first, all instructions in the basic blocks are duplicated except branch or jump instructions. This is due to duplication of branch/jump instruction may cause infinite loop or program hang. Next, a comparison instruction is placed after the original and the duplicated instruction to compare their results [7]. If any mismatch is detected, an error is reported. By duplicating the entire code base except for branch/jump instructions with $E D^{4} I$, good fault detection ratio at the expense of execution time and code size usage can be achieved.

\section{B. Selective Code Duplication}

In selective code duplication techniques, only a part of the code is duplicated and compared, this results in reduced fault detection ratio with improvement in overhead. Most of the proposed selective code duplication techniques were based on a wide variety of possibilities to identify the errorprone blocks in a control flow graph (CFG). The CFG is a representation of the program and its flow, in which the program is divided into a number of basic blocks and edges. A basic block is a sequence of consecutive instructions with exactly one entry and one exit point [23], [24]. An edge is intentional path between the basic blocks based on branch and jump instructions presented at the end of basic blocks.

From this selective code duplication category, we selected CBD [18] and SWIFT [6] techniques to compare with our FDSC technique. This is because they proved to be the best techniques for achieving a better fault detection ratio among other selective code duplication mechanisms as can

Table I

EXAMPLE FOR ASSEMBLY-LEVEL CODE DUPLICATION AND COMPARISON

\begin{tabular}{|c|c|}
\hline Unprotected Assembly-Level Code & Protected Assembly-Level Code \\
\hline add r3, r3, r2 & add r3, r3, r2 \\
& add r3, r3', r2' \\
cmp r3, r3' & bne error \\
ldr r2, [r3, r0] & ldr r2, [r3, r0] \\
& ldr r2', [r3', r0'] \\
cmp r2, r2' & bne error \\
& str r1, [r2, r0] \\
str r1, [r2, r0] & str r1', [r2', r0'] \\
& cmp r1, r1' \\
& bne error \\
& mov r4, r1 \\
mov r4, r1 & mov r4', r1' \\
& cmp r4, r4' \\
& bne error \\
& cmp r3, r3' \\
& bne error \\
cmp r2, r2' & bne error \\
& cmp r3, r2 \\
& ble loop \\
\hline ble loop & \\
\hline
\end{tabular}


be seen in [20]. There exist more selective code duplication techniques than full code ones in the literature [14]. That motivated us to consider two of the best ones from this category for comparison.

The core idea behind $\mathrm{CBD}$ is to only duplicate and insert comparison instructions in critical basic blocks to check for errors. Abdi, Asghari, Pourmozaffari, Taheri and Pedram [18] define a critical basic block as the basic block with the highest fan out, because such blocks can impact many other basic blocks.

SWIFT is a selective code duplication mechanism based on instructions. In this technique, all instructions in the basic blocks, except store instructions, are duplicated. The basic idea involved in skipping those store instructions from duplication is to avoid creating multiple memory locations that further avoids extra overhead [6]. Comparison instructions are placed before store and branch instructions to compare the results. In this way, overheads are improved in SWIFT.

\section{FDSC TECHNIQUE}

As aforementioned, our FDSC technique aims to merge the advantages of both full code and selective code duplication techniques. As the name gives away, we opt to duplicate the entire code base of the original program and place the necessary comparison instructions in a few selected critical basic blocks only. The critical basic blocks in which the comparison instructions are inserted are basic blocks with two or more incoming edges. Such basic blocks are more likely to be included in many execution paths throughout the target algorithm. This means that a maximum of errors can be detected with a minimum of comparison instructions.

Fig. 1 explains why we opted to duplicate the entire code base instead of only duplicating the basic blocks which have a comparison instruction inserted. The left-hand side shows a selective duplication technique in which only the comparison basic block has its code duplicated. In contrast, the righthand side shows our FDSC technique implemented for the same CFG. In both parts, the critical basic block is indicated by a light grey fill.

As can be seen on the left-hand side of Fig. 1, when implementing a selective code duplication technique, the shadow registers (indicated with ') are only filled and used in the critical basic block. This leaves room for errors to go unnoticed in the non-critical basic blocks as their operations are not duplicated on the shadow registers and their results cannot be validated. We have indicated those basic blocks in red. In essence, only one out of the four basic blocks in the shown CFG are now protected.

When applying our FDSC technique, thus applying full code duplication, the operation of the non-critical basic blocks can be verified in the critical basic blocks. The shadow registers are updated and can be used to verify the results of the operations. Therefore, three out of the four shown basic blocks are now protected and indicated

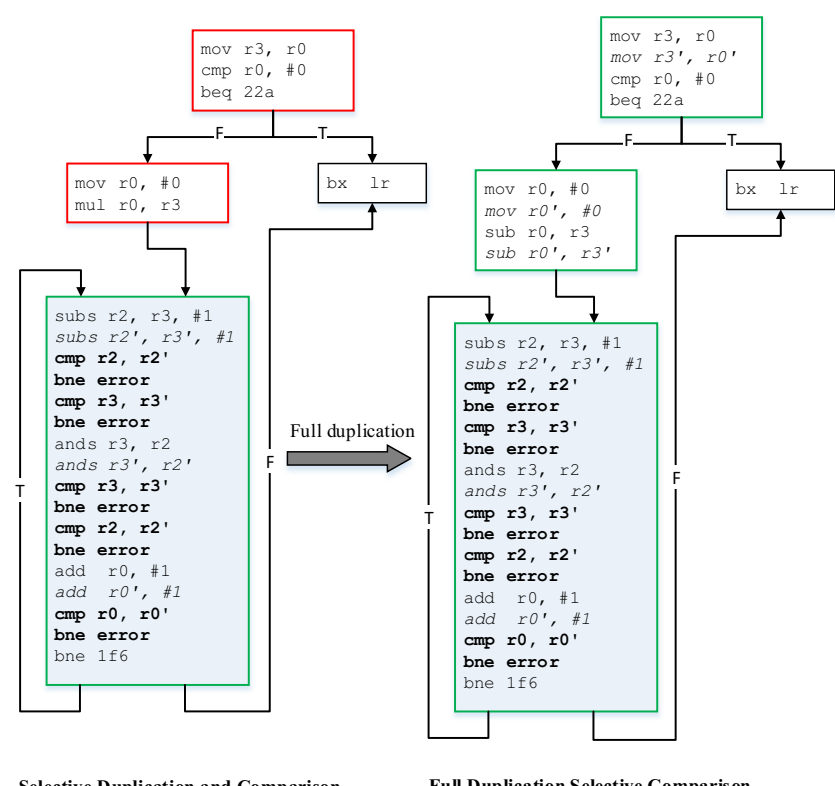

Selective Duplication and Comparison

Full Duplication Selective Comparison

Fig. 1. Example for CFG of the sample assembly-level code to explain the advantage of FDSC.

in green. To conclude, the full code duplication allows us to protect a large number of the basic blocks in the CFG of the targeted program. The selective insertion of the comparison instructions aids in keeping the imposed overhead as low as possible.

\section{EXPERIMENTAL SETUP}

This section describes the components used for the experiments. We first start with describing which case studies were considered. Next, we present the hardware and fault injection tool was used to test the implemented data error detection techniques. In the end, we discuss criteria used in order to evaluate all described techniques.

\section{A. Case Studies}

To validate the newly proposed FDSC and compare it to the $E D^{4} I, \mathrm{CBD}$ and SWIFT techniques, we first implemented them for the seven popular case studies. These programs are the implementation of bubble sort (BS), quicksort (QS), matrix multiplication (MM), bit count (BC), cyclic redundancy check (CRC), insertion sort (IS) and selection sort (SS) algorithms. Being highly used as validation case studies in the published literature for the data error detection techniques, these case studies also have varying complexity and CFGs as shown by Vankeirsbilck, Penneman, Hallez and Boydens [23]. This makes them highly suitable for our experiments.

Moreover, different sorting algorithms were selected due to the sorting of information is used in different applications, 
e.g., to perform a big data analysis and interpretation, one needs to rely on priorities to perform fast analysis [25], [26]. The MM algorithm was selected because it is used in various embedded domains such as image processing and compression, robotics [27]. The extensive application based overview of BC and CRC and its usage is well explained by Guthaus et al. [25]. Among the seven considered case studies, BC and CRC are from the MiBench [25].

\section{B. Hardware}

The selected case studies were executed on a NXP LPC1768, an ARM Cortex-M3 driven microcontroller. The ARM Cortex-M3 was selected because it is an industry lead-

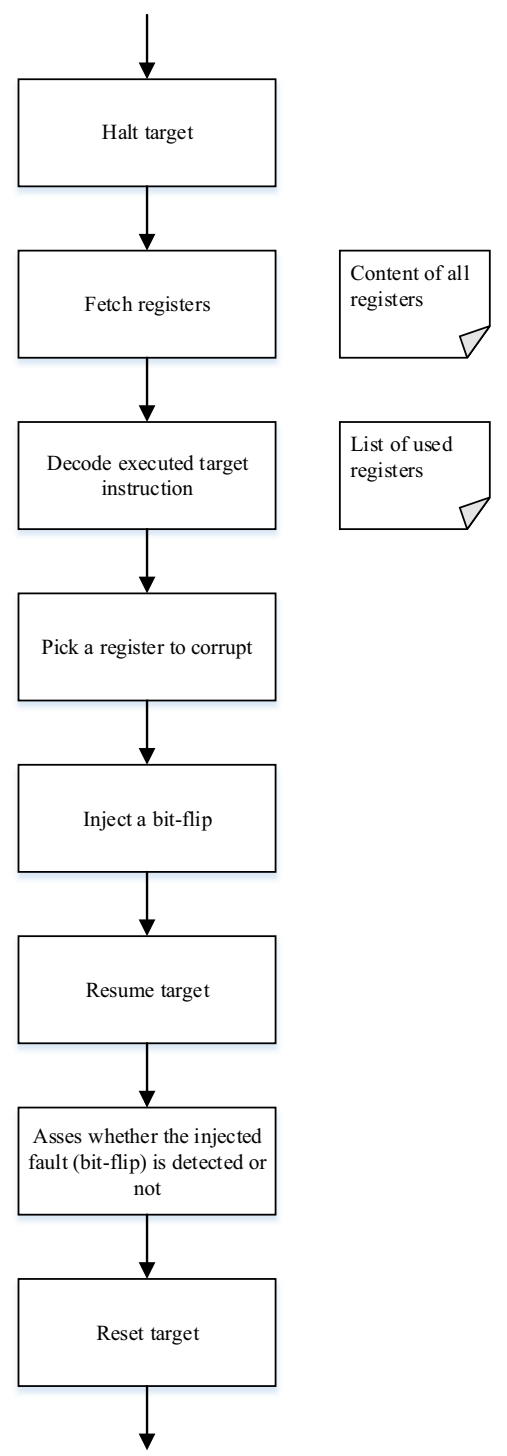

Fig. 2. Fault injection process using SWIFI tool. ing 32-bit processor used in various embedded application domains [28].

\section{Fault Injection Process and Tool}

To test the implementations, data errors need to occur in the target system. To make them occur, we inject bitflips using our in-house fault injection tool. It is known that most systems already have some countermeasures in place for their memory like parity and ECC but not for CPU registers [29]. For this reason, we only inject bit-flips in CPU registers to validate the capabilities of our FDSC technique. To deterministically and directly inject data errors into the processor registers, the instruction aware injection mechanism was used [30]. The fault injection process per bit-flip according to the instruction aware injection process is shown in Fig. 2. In Fig. 2, decoding the executing target instruction and then identifying the corresponding registers is the critical step in the fault injection process. Once the list of used registers are identified, it is easy to corrupt the information in those registers by flipping a bit. As soon as the bit is flipped, the target is resumed for further fault injections.

In addition, we have integrated instruction aware injection mechanism into our in-house developed softwareimplemented fault injection (SWIFI) tool. SWIFI is a known method to validate fault tolerance techniques. This tool was also used to validate the implemented techniques in our previous publications [11], [20], [23]. Our tool injects data flow errors at runtime. The SWIFI tool focuses on the ARM Cortex-M processor family [28], [31]. Fig. 3 shows the architectural overview of the SWIFI tool. To access the target device, the SWIFI tool uses the Cortex microcontroller software interface standard debug access port (CMSIS-DAP) interface of the Cortex-M3 processor [28]. This interface gives us access to the on-chip debugger, which allows us to access and manipulate the information in all the registers of the target device. In Fig. 3, the external host PC is connected to the target and the CMSIS-DAP interface using universal serial bus (USB) to inject bit-flips.

The advantage of a SWIFI tool over other fault injection mechanisms is that it deterministically injects faults with minor randomness, leading to a very good fault injection rate in the target system. Next, we injected 3200 data errors per case study for validation. We also validated by injecting 320 data errors per case study that resulted in the same detection

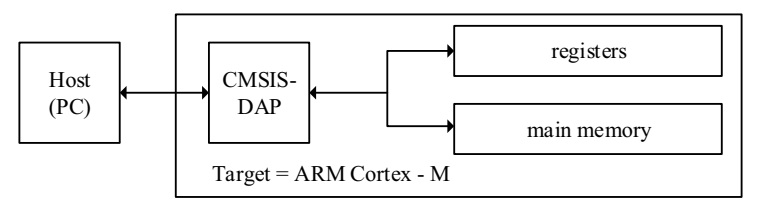

Fig. 3. SWIFI tool architecture. 
ratio as with 3200 faults.

After validating fault injection, obtained results are analyzed based on the following classification:

Software Detected Fault (SDF): The injected fault is detected by the duplication and comparison instructions used for data error detection.

Hardware Detected Fault (HDF): The injected fault is detected by the default hardware fault exception handler in ARM Cortex processor family.

Silent Data Corruption (SDC): The injected fault is not detected and it changes the output of the program.

No Effect Fault (NEF): The injected fault does not have an effect on the output of the program.

\section{Evaluation Criteria}

In this part, we discuss the three evaluation criteria measured for each technique: fault detection ratio (FDR), code size overhead (CSO) and execution time overhead (ETO). To record the fault detection ratio, we used (1). Equation (1) counts the malicious faults that were detected by the implemented countermeasure. However, fault detection does not come without a cost, as both duplication and comparison mechanisms introduce code size and execution time overheads. Code size overhead indicates the increase in the size of the protected code, whereas execution time overhead indicates the extra time it takes to execute the protected code. Code size overhead and execution time overhead are calculated using (2) and (3). Moreover, in (1), (2) and (3), SDF indicates software detected faults, TIF indicates total injected faults, NEF indicates no effect faults, PCS indicates protected code size, UPCS indicates unprotected code size, PET indicates protected execution time and UPET indicates unprotected execution time.

$$
\begin{aligned}
\mathrm{FDR} & =\frac{\mathrm{SDF}}{\mathrm{TIF}-\mathrm{NEF}} \\
\mathrm{CSO} & =\frac{\text { PCS }-\mathrm{UPCS}}{\mathrm{UPCS}} \\
\mathrm{ETO} & =\frac{\text { PET }-\mathrm{UPET}}{\mathrm{UPET}}
\end{aligned}
$$

\section{RESUlts AND Discussion}

This section discusses the results of our experiments. We compare the results of our technique to $E D^{4} I, \mathrm{CBD}$ and SWIFT techniques. First, we show the results of the performed fault injection using the four defined fault categories. Next, we compare the techniques based on the three evaluation criteria discussed in the previous section.

Fig. 4 shows the results we obtained from validation in the form of SDF, HDF, SDC and NEF. Analyzing Fig. 4, $E D^{4} I$ which is a full code duplication technique, has the highest percentage of SDF for most considered case studies. In addition to the SDF, the percentage of faults detected by HDF category is much lower. However, our FDSC is a software-based technique, so we pay more attention to the faults detected by FDSC and less to the faults detected by default hardware-based fault handlers. The third category contains SDC: in $E D^{4} I$, case studies on the third category showed very low SDC percentages. This is because the entire code base is protected using duplication and comparison. The final fault category is referred to as NEF. This category represents the faults which do not have an effect on the output of the program. Therefore, we make comparisons based on the SDF and SDC.

In contrast to $E D^{4} I$, the selective code duplication techniques $\mathrm{CBD}$ and SWIFT generate the lowest percentage of SDF for almost all case studies. On the other hand, the percentage of SDC per case study is higher as a result of their selective duplication and comparison mechanism. In this case, the achieved results reflect the theory behind selective code duplication mechanisms which is contrast to the full code duplication mechanisms.

In addition, closer to $E D^{4} I$ is our FDSC technique, which is a combination of both full duplication and selective comparison mechanisms. The percentage of SDF in our technique is almost similar to the $E D^{4} I$ because of its full duplication. On the other hand, compared to CBD and SWIFT, the percentage of SDF in FDSC improved for all case studies. This is the result of duplicating the entire code base like in $E D^{4} I$ and of placing comparison instructions only in the most vulnerable blocks.

\section{A. Fault Detection Ratio}

Fig. 5 shows the calculated values of the fault detection ratio. Analyzing Fig. 5, it can be seen that $E D^{4} I$ has the highest fault detection ratio among most case studies and also a higher average percentage. On the other hand, in comparison with $E D^{4} I$, fault detection ratio in CBD and SWIFT are reduced by $20 \%$ and $59 \%$ respectively, because of protecting only a selective part of the code. In SWIFT technique, fault detection ratio for BC algorithm has reduced by a big margin. This is the result of the position of comparison instructions. In detail, the $\mathrm{BC}$ algorithm does not contain store instructions to perform comparisons before them. For this reason, comparisons were performed only before branch instructions, which cause a reduction in fault detection ratio.

Moreover, in FDSC, the fault detection ratio was comparatively close to $E D^{4} I$, because of its full code duplication. The aim of our technique is to overcome the drawbacks from the considered full and selective code duplication techniques. The fault detection ratio of our FDSC is improved by $14.2 \%$ and $51.8 \%$, respectively in comparison with CBD and SWIFT. The achieved results by merging the ideas of full code and selective code duplication mechanisms clearly indicate the advantage of our FDSC technique with respect to fault detection capabilities. 


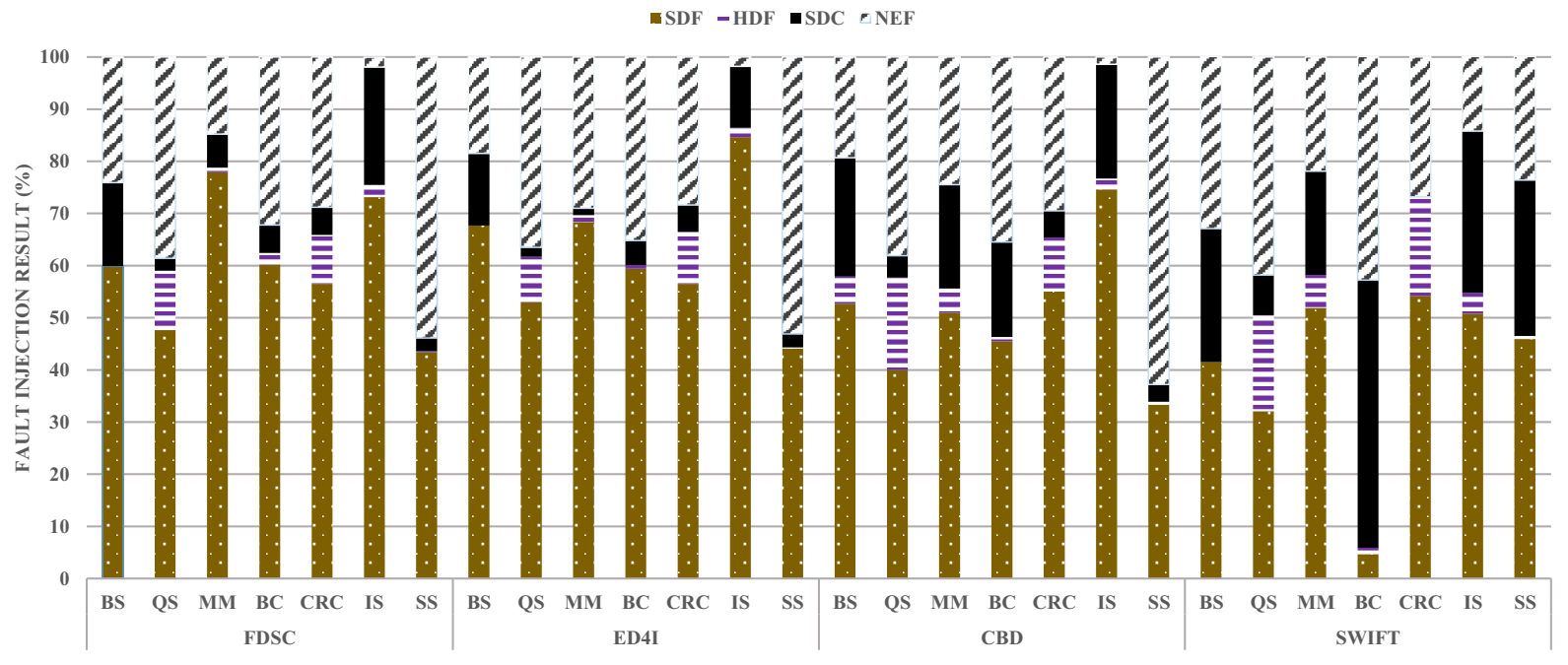

Fig. 4. Fault injection results of FDSC, $E D^{4} I, \mathrm{CBD}$ and SWIFT techniques.

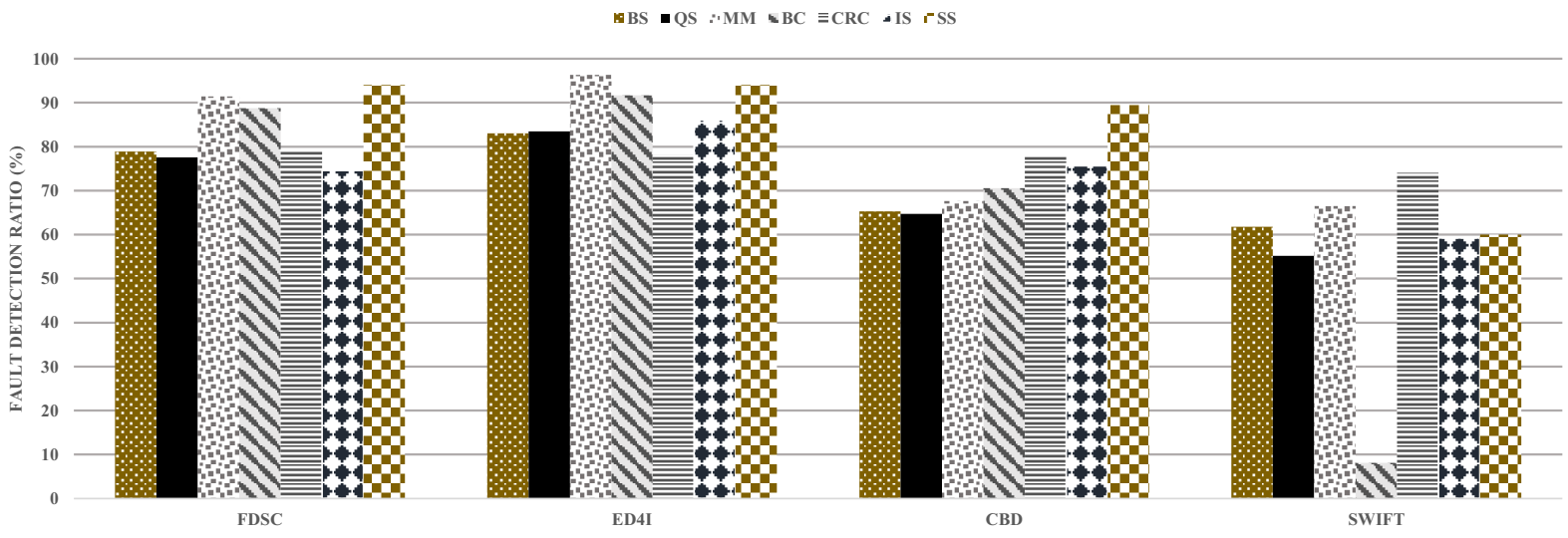

Fig. 5. Fault detection ratio of FDSC, $E D^{4} I, \mathrm{CBD}$ and SWIFT techniques.

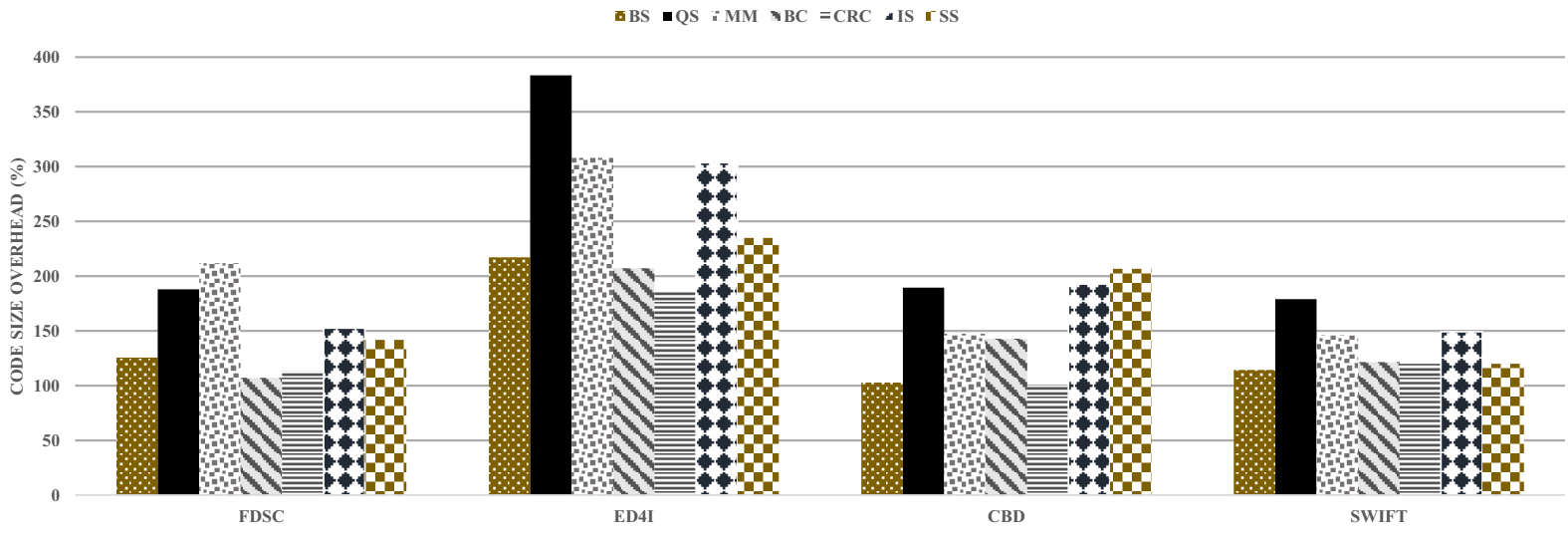

Fig. 6. Code size overhead of FDSC, $E D^{4} I, \mathrm{CBD}$ and SWIFT techniques. 


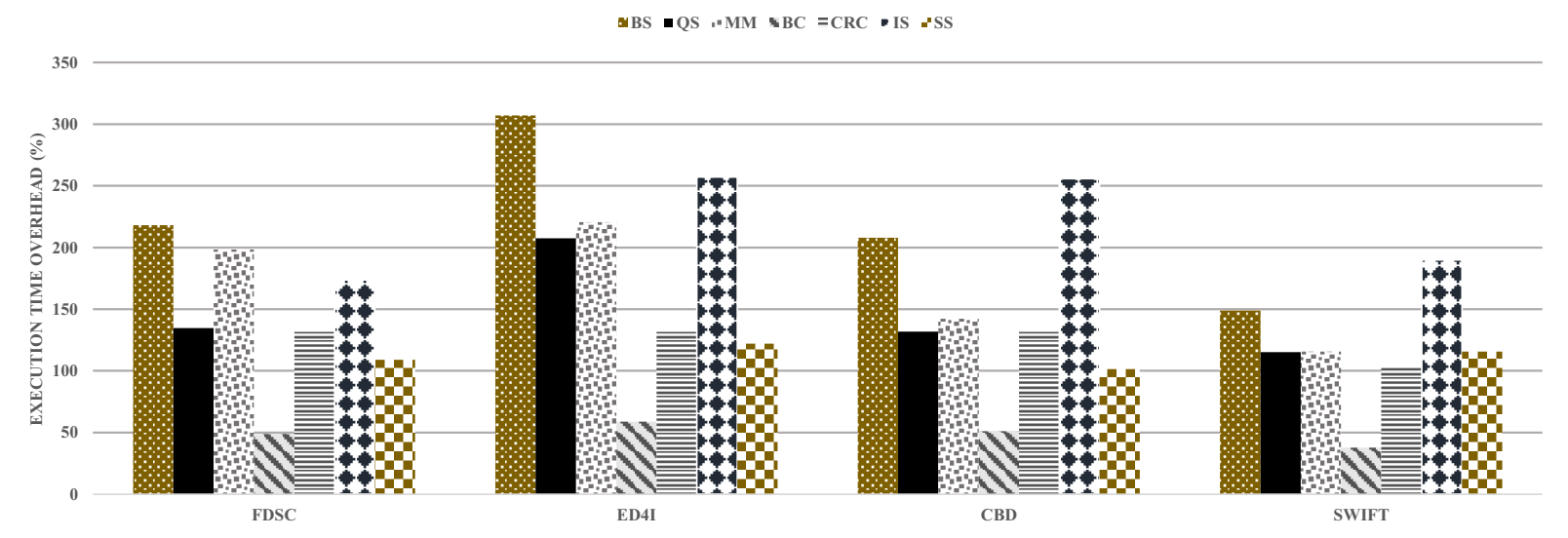

Fig. 7. Execution time overhead of FDSC, $E D^{4} I, C B D$ and SWIFT techniques.

\section{B. Code Size Overhead}

Code size overhead for the different case studies and different techniques are shown in Fig. 6. They are calculated based on the number of duplication and comparison instructions added to the original code. Examining Fig. 6, it can be seen that FDSC, CBD and SWIFT have nearly equal code size overheads among most case studies. In comparison with $E D^{4} I$, results show that code size overhead of our FDSC is improved by $43.5 \%$. This is because FDSC requires less comparison instructions than $E D^{4} I$. In $E D^{4} I$, despite having a good fault detection capabilities, the introduced code size overheads are very high.

\section{Execution Time Overhead}

Execution time overheads are given in Fig. 7. Evaluating Fig. 7, it is clear that FDSC and CBD again nearly have the same lower percentage of execution time. In comparison with SWIFT, results show that execution time overhead of FDSC has reduced by $22.9 \%$. Similarly, as in code size overhead, comparison with $E D^{4} I$, results showed that execution time overhead of FDSC has improved by $22.2 \%$. To conclude, Figs. 5, 6, and 7 show that it pays off to merge the ideas behind full code and selective code duplication to benefit from their advantages. The advantage of the proposed FDSC clearly indicates that a higher fault detection ratio with a lower overhead is possible.

\section{CONCLUSION}

Initially, we mentioned and explained three considered data error detection techniques used for obtaining a comparison. One is from full code duplication mechanisms; $E D^{4} I$ and the other two are from selective code duplication mechanisms; CBD and SWIFT. It is clear from the results that $E D^{4} I$ provide better fault detection ratio at the expense of execution time and code size usage. In contrast, CBD and SWIFT provide better overheads with a reduction in fault detection ratio.
In this paper, we presented a new data error detection technique based on software redundancy and instruction duplication. The aim of our technique was to improve the fault detection ratio in selective code duplication techniques and code size overhead and execution time overhead for full code duplication techniques. In the proposed FDSC technique, the entire code base except branch instructions are duplicated and comparison instructions are placed only in the vulnerable blocks. This is to avoid extra overhead. Compared to $E D^{4} I$, results have shown that the code size overhead and execution time overhead of FDSC improved by $43.5 \%$ and $22.2 \%$, respectively, with only $4.5 \%$ reduction in fault detection ratio. On the other hand, in comparison with the average of CBD and SWIFT, results have shown that the fault detection ratio of FDSC improved by $33 \%$ with only $2.8 \%$ and $10.9 \%$ reduction in code size and execution time overhead respectively. The advantage of our FDSC technique is that it clearly addresses and overcomes the drawbacks in full and selective code duplication mechanisms. The results achieved with the proposed FDSC technique have an added value to the full code and selective code duplication techniques existing in the state-of-the-art in the literature.

\section{FUTURE WORK}

Currently, our research focuses on data error detection. In fault-tolerant embedded systems, error detection by itself is not enough to make them fault-tolerant. It also needs error recovery. Thus, our future work will focus on this addition. In more detail, error recovery is generally based on the checkpointing concept. Checkpoints are saved at regular intervals to check for irregular behavior. Such techniques are further classified into rollback error recovery and rollforward error recovery techniques. We will use one of the potential checkpointing mechanism on top of our error detection mechanism to recover the errors. 


\section{REFERENCES}

[1] S. A. Asghari, A. Abdi, H. Taheri, H. Pedram, and S. Pourmozaffari, "SEDSR: Soft error detection using software redundancy," Journal of Software Engineering and Applications, vol. 5, no. 09, pp. 664-670, 2012.

[2] J. A. Martínez, J. A. Maestro, and P. Reviriego, "Evaluating the impact of the instruction set on microprocessor reliability to soft errors," IEEE Transactions on Device and Materials Reliability, vol. 18, no. 1, pp. 70-79, 2018.

[3] R. C. Baumann, "Soft errors in advanced semiconductor devices-part i: the three radiation sources," IEEE Transactions on device and materials reliability, vol. 1, no. 1, pp. 17-22, 2001.

[4] T. Karnik and P. Hazucha, "Characterization of soft errors caused by single event upsets in cmos processes," IEEE Transactions on Dependable and Secure Computing, vol. 1, no. 2, pp. 128-143, 2004.

[5] S. Jagannathan, Z. Diggins, N. Mahatme, T. Loveless, B. Bhuva, S. Wen, R. Wong, and L. Massengill, "Temperature dependence of soft error rate in flip-flop designs," in IEEE International Reliability Physics Symposium (IRPS). IEEE, 2012, pp. SE2.1-SE2.6.

[6] G. A. Reis, J. Chang, N. Vachharajani, R. Rangan, and D. I. August, "SWIFT: Software implemented fault tolerance," in Proceedings of the international symposium on Code generation and optimization. IEEE Computer Society, 2005, pp. 243-254.

[7] N. Oh, S. Mitra, and E. J. McCluskey, "ED4I: error detection by diverse data and duplicated instructions," IEEE Transactions on Computers, vol. 51, no. 2, pp. 180-199, 2002.

[8] B. Arasteh, A. Bouyer, and S. Pirahesh, "An efficient vulnerability-driven method for hardening a program against soft-error using genetic algorithm," Computers \& Electrical Engineering, vol. 48, pp. 25-43, 2015.

[9] M.-L. Li, P. Ramachandran, S. K. Sahoo, S. V. Adve, V. S. Adve, and Y. Zhou, "SWAT: An error resilient system," Proceedings of SELSE, 2008.

[10] J. Vankeirsbilck, H. Hallez, and J. Boydens, "Soft error protection in safety critical embedded applications: An overview," in 10th International Conference on P2P, Parallel, Grid, Cloud and Internet Computing (3PGCIC). IEEE, 2015, pp. 605-610.

[11] J. Vankeirsbilck, V. B. Thati, H. Hallez, and J. Boydens, "Inter-block jump detection techniques: A study," in International Scientific Conference Electronics (ET). IEEE, 2016, pp. 1-4.

[12] N. Oh, P. P. Shirvani, and E. J. McCluskey, "Control-flow checking by software signatures," IEEE transactions on Reliability, vol. 51, no. 1, pp. 111-122, 2002.

[13] A. Abdi, S. A. Asghari, S. Pourmozaffari, H. Taheri, and H. Pedram, "An effective software implemented data error detection method in real time systems," in Advances in Computer Science, Engineering \& Applications. Springer, 2012, pp. 919-926.

[14] V. B. Thati, J. Vankeirsbilck, J. Boydens, and D. Pissoort, "Data error detection and recovery in embedded systems: a literature review," Advances in Science, Technology and Engineering Systems Journal, vol. 2, no. 3, pp. 623-633, 2017.

[15] S. Feng, S. Gupta, A. Ansari, and S. Mahlke, "Shoestring: probabilistic soft error reliability on the cheap," in $A C M$ SIGARCH Computer Architecture News, vol. 38, no. 1. ACM, 2010, pp. 385-396.
[16] E. Chielle, F. Rosa, G. S. Rodrigues, L. A. Tambara, F. L. Kastensmidt, R. Reis, and S. Cuenca-Asensi, "Reliability on arm processors against soft errors by a purely software approach," in 15th European Conference on Radiation and Its Effects on Components and Systems (RADECS). IEEE, 2015, pp. 1-5.

[17] B. Nicolescu and R. Velazco, "Detecting soft errors by a purely software approach: method, tools and experimental results," in Embedded Software for SoC. Springer, 2003, pp. $39-51$.

[18] A. Abdi, S. Asghari, S. Pourmozaffari, H. Taheri, and H. Pedram, "An optimum instruction level method for soft error detection," International Review on Computers and Software, vol. 7, no. 2, 2012.

[19] A. Li and B. Hong, "Software implemented transient fault detection in space computer," Aerospace science and technology, vol. 11, no. 2-3, pp. 245-252, 2007.

[20] V. B. Thati, J. Vankeirsbilck, N. Penneman, D. Pissoort, and J. Boydens, "CDFEDT: Comparison of data flow error detection techniques in embedded systems: an empirical study," in Proceedings of the 13th International Conference on Availability, Reliability and Security. ACM, 2018, pp. 23:1-23:9.

[21] N. Oh, P. P. Shirvani, and E. J. McCluskey, "Error detection by duplicated instructions in super-scalar processors," IEEE Transactions on Reliability, vol. 51, no. 1, pp. 63-75, 2002.

[22] B. Nicolescu, Y. Savaria, and R. Velazco, "Software detection mechanisms providing full coverage against single bit-flip faults," IEEE Transactions on Nuclear science, vol. 51, no. 6, pp. 3510-3518, 2004.

[23] J. Vankeirsbilck, N. Penneman, H. Hallez, and J. Boydens, "Random additive signature monitoring for control flow error detection," IEEE Transactions on Reliability, vol. 66, no. 4, pp. 1178-1192, 2017.

[24] F. E. Allen, "Control flow analysis," in ACM Sigplan Notices, vol. 5, no. 7. ACM, 1970, pp. 1-19.

[25] M. R. Guthaus, J. S. Ringenberg, D. Ernst, T. M. Austin, T. Mudge, and R. B. Brown, "Mibench: A free, commercially representative embedded benchmark suite," in IEEE International Workshop on Workload Characterization, WWC-4. IEEE, 2001, pp. 3-14.

[26] R. Zhu and Y. Ma, "Information engineering and applications," vol. 5, no. 7. International Conference on Information Engineering and Applications (IEA), 2011.

[27] R. C. Gonzalez and R. E. Woods, "Digital image processing," 2012.

[28] Cortex-m3 processor' arm. [Online]. Available: https://developer.arm.com/products/processors/cortex$\mathrm{m} /$ cortex-m3

[29] S. S. Mukherjee, J. Emer, T. Fossum, and S. K. Reinhardt, "Cache scrubbing in microprocessors: Myth or necessity?" in Proceedings. 10th IEEE Pacific Rim International Symposium on Dependable Computing. IEEE, 2004, pp. 37-42.

[30] A. Heinig, I. Korb, F. Schmoll, P. Marwedel, and M. Engel, "Fast and low-cost instruction-aware fault injection." in GIJahrestagung, 2013, pp. 2548-2561.

[31] K. Norman, "Integrating web servers in embedded applications," Luminary Micro Applications Engineer, Luminary MicroWild Basin, Suite, vol. 350, 2007. 